\title{
Trends in malaria cases and stratification of malaria incidence in the malaria elimination setting, Harari Region, Eastern Ethiopia
}

Endashaw Esayas ( $\nabla$ esu.endo@gmail.com )

Armauer Hansen Research Institute https://orcid.org/0000-0001-7116-7033

Asefa Tufa

Harari Region Health Bureau

Fekadu Massebo

Arba Minch University

Abdulhamid Ahemed

Harari Region Health Bureau

Ibssa Ibrahim

Harari Region Health Bureau

Dereje Dillu

Federal Ministry of Health

Eyuel Asemahegn Bogale

Armauer Hansen Research Institute

Solomon Yared

Jigjiga University

Kebede Deribe

Addis Ababa University School of Public Health

\section{Research}

Keywords: Elimination, Harari region, Ethiopia, Incidence, Malaria, Sub-district, Stratification

Posted Date: June 12th, 2020

DOl: https://doi.org/10.21203/rs.3.rs-34668/v1

License: (1) (i) This work is licensed under a Creative Commons Attribution 4.0 International License.

Read Full License 


\section{Abstract}

Abstract Background: Ethiopia has shown a notable progress in reducing the burden of malaria over the last two decades. Based on the progress, the country shifted from control to elimination of malaria. This study was conducted to analyse trends in malaria cases and stratification of malaria incidence in the malaria elimination setting in eastern Ethiopia. Methods: A retrospective malaria data recorded from 2013 to 2019 were reviewed from Harari Region, eastern Ethiopia. In addition, three years malaria data were used to assess the sub-district (kebele) level stratification of malaria incidence. Results: A total of 44,882 (46.9\%) malaria cases were detected from 95,629 malaria-suspected outpatient diagnosed in Harari Region from 2013 to 2019. Of these, 41,046 were confirmed malaria cases (microscopically and rapid diagnostic test) while 3,836 were reported as clinical cases. In the region, malaria trend was fluctuating year to year, the high peak was reported in 2016 but malaria cases showed decreasing trend in number of malaria cases from 2017 to 2019. Plasmodium falciparum , P. vivax and mixed infections were accounted for $69.2 \%, 30.6 \%$ and $0.2 \%$ of the cases, respectively. The malaria risk appears to be heterogeneous and varies between districts, higher number of malaria cases were recorded in Jenella, Erer and Amir Nur districts, and about $80 \%$ of the cases were from these districts. According to the latest (2019) sub-district ( kebele ) level epidemiological data of malaria stratification, $8.3 \%$ of the sub-districts in the Harari region reported no malaria and a majority (61.1\%) of sub-districts reported fewer than five cases per thousand population. Furthermore, there were no high malaria strata in the Region. The highest peak of malaria cases in the Region was reported from September to November followed by from April to May. Conclusions: In the Harari Region, the retrospective malaria data showed a significant declining trend. Thus, if this achievement is sustained and scaling-up of the existing malaria prevention and control strategies by focusing on those populations living in the higher malaria transmission districts and subdistricts, planning of malaria elimination from the study area might be feasible. Key words: Elimination, Harari region, Ethiopia, Incidence, Malaria, Sub-district, Stratification

\section{Background}

Malaria poses threats to the public health of the world, with particular burden of diseases in sub-Saharan Africa [1]. Globally, there were an estimated 228 million new malaria cases and of these about 405,000 malaria-related deaths in 2018, and mostly the deaths are common in children under the age of five years in sub-Saharan Africa. The world health organization (WHO) African Region bearing the highest burden of malaria cases (93\%) and deaths (94\%) [2].

In Ethiopia, the burden of malaria continues to cause a substantial number of morbidity and mortality which accounts for most outpatient visits, and it has been one of the main causes of hospitalization and deaths in the country $[3,4]$. About $60 \%$ of the population are living in the malaria risky areas mainly areas that lie below 2000 meters above sea level $[4,5]$. However, several pockets with micro-epidemiological conditions supporting malaria transmission occur in areas above this altitude [6, 7]. $P$. falciparum and $P$. vivax are the dominant parasites responsible for the majority of malaria cases in Ethiopia. The contribution of the other Plasmodium species in the country is negligible $[5,8]$. 
Since the 2000 there has been substantial increase in the investment to support malaria interventions in Africa including Ethiopia. Due to this commitment in the last two decades significant reduction of malaria has been registered in Ethiopia. This improvement could be attributed to improved coverage of key antimalarial interventions, including, distribution of long-lasting insecticidal nets through mass campaigns targeting the entire population at risk, indoor residual spraying (IRS) in designated epidemicprone areas, and expanded diagnostic testing and effective antimalarial treatment to people at risk $[5,8$, $9,10]$. Thus, based on this progress Ethiopia has recently planned to achieve the nationwide malaria elimination by 2030 by starting sub-national elimination in districts with low malaria transmission [8].

Malaria trend analysis is used for public health surveillance and monitoring, forecasting, program evaluation, policy analysis and for etiologic analysis (investigation of potentially causal relationships between risk factors and outcomes) $[3,11]$. Thus, analysing malaria prevalence trends and the intervention employed each year are important for the expansion of intervention strategies or to design new ones to tackle the disease. Harari Region was selected by the National Malaria Elimination Program for the possible malaria elimination and as such, a malaria elimination strategy was launched and is under implementation. However, the overall trend of malaria cases is not studied in the Region. Therefore, this study was aimed at analyzing the seven-year trend of malaria cases in the Harari Region.

Furthermore, the present study used to determine the malaria incidence stratification at sub-district and district levels, which may contribute to the evidence-based decision for malaria control and elimination strategies in the Region.

\section{Methods}

\section{Study area description}

This study was carried out in Harari Regional State, one of the Regional States of Federal Democratic Republic of Ethiopia, is located in the eastern part of the country 515 kilometres from the national capital Addis Ababa. The region is surrounded by Oromia Regional State (Fig. 1). The Region covers a total area of 342.2 square kilometres with the estimated total population of 302,000 . The region located within $9^{\circ} 11^{\prime} 49^{\prime \prime}-9^{\circ} 24^{\prime} 42^{\prime \prime} \mathrm{N}$ Latitude and $42^{\circ} 03^{\prime} 30^{\prime \prime}-42^{\circ} 16^{\prime} 24^{\prime \prime} \mathrm{E}$ Longitude and the altitude of the Region ranges between 1552 and 1957 meters above sea level. The mean annual temperature of the Region ranges from $18-21^{\circ} \mathrm{C}$ and mean annual rainfall ranges from $687-775$ millimetres. The Region is divided into three rural districts (Dire Teyara, Sofi and Erer districts) and 6 urban districts within Harar Town Administration (Abadir, Aboker, Amir Nur, Jenella, Hakim and Shenkor districts). These districts are administratively further sub-divided into 36 sub-districts (kebeles). Since 2016, the potential primary health service in the Region is estimated to be about $100 \%$. In Harari Regional State there are six hospitals (two governmental, two military, a private and a non-governmental), one Regional laboratory and research centre, 8 health centres, 32 health posts and 40 private clinics [12]. In addition to the regional people, the health facilities in the region are giving service for the eastern part of Ethiopia. For 
instance, the Regional laboratory is serving as testing centre for early infant diagnosis for health facilities of eastern Hararge zone of Oromia Region, Dire Dawa city administration and Somali Region.

\section{Study design}

A retrospective study was conducted to determine seven years (from January, 2013 to December, 2019) malaria case trends by reviewing the total malaria data of the district health offices. In addition, 3-years (from January, 2017 to December, 2019) malaria data was used to assess the sub-district level stratification of malaria incidence.

\section{Malaria case and data management in the Harari Region}

In the Harari Region malaria is diagnosed clinically and parasitological. The clinical diagnosis is primarily considering fever or a history of fever (a febrile illness) with or without other symptoms that is suspected by a health worker as malaria infection. Whereas parasitological diagnosis is confirmation of malaria cases by using a diagnostic test (light microscopy [gold standard] or rapid diagnostic tests [RDTs]) [10, 13]. Malaria services such as malaria testing, treatments, distribution of nets are rendered in all health facilities (health post, health centers and hospitals) in the Region. In the health post patients are treated clinically or using RDTs. The health posts report malaria case data monthly to their nearest health centre health management information system office (HMIS). In the Region each health centre has on average four health posts. In addition, confirmed malaria cases are reported monthly from laboratory unites to the corresponding health centre and hospital HMIS. The Region strictly follows the standard operating procedures (SOPs) in all phases of the quality control. In the region, peripheral smear examination of a well-prepared and well-stained blood film is used as the gold standard in confirming the presence of the malaria parasite as per the WHO protocol [13]. All hospitals and health centres in the Region follow a SOP for capillary blood sample collection, smear preparation, staining techniques and blood film examination for malaria parasite detection. Finally, all malaria cases data were reported monthly from each health centre and hospitals to the district HMIS. Data were stored electronically in HMIS of the district by month, year, clinically treated, RDT and microscopically confirmed cases. The present study included all malaria cases data recorded between 2013 and 2019 of the Harari Regional State.

\section{Data collection techniques}

A format was prepared to collect the secondary data from the districts and Regional database. With the help of the HMIS expert, individual data such as total clinically treated, and confirmed cases in month and year, types of malaria species and address of patient were collected from the recorded sheet. During data collection, the data were strictly checked for its completeness and any data such as address of patient, and malaria diagnosis result which is not properly recorded was excluded from the analysis. In this record review a very small number (only eight individual data) were excluded due to incompleteness of registrations that miss either address of patient or malaria diagnosis result (types of malaria species).

\section{Data analysis}


Data were first entered into Excel and then imported into IBM® SPSS ${ }^{\circledR}$ version 20 (Armonk, New York: IBM Corporation) for analysis. Descriptive statistics was used to show the trends of malaria transmission in terms of seasons, years and species of malaria parasite. The Pearson's Chi square test was used to describe the associations of variables. Test of significance was estimated assuming a at 0.05 and a pvalue less than 0.05 was considered significant. Finally, the retrospective malaria data were described and presented with appropriate figures and tables. The annual parasite incidence (API) per 1000 population at risk was computed using the total population of each year as a denominator and confirmed cases of malaria as a numerator. API was calculated based on the current sub-district population, 2007 Ethiopian government census and 2013 projections at district and regional levels assuming constant population growth rate during the study period. Numbers of districts with malaria-free, low, moderate and high API were calculated separately for 2019. The total population and percentage composition in different transmission categories was calculated accordingly. Malaria positivity rate was calculated as number of confirmed cases over the total suspected febrile case examined at study sub-districts. Seasonality was determined by monthly positivity rate of total infections and by species. Species composition was calculated annually. Finally, all maps were produced using ArcGIS Desktop v10.5 (Environmental Systems Research Institute Inc., Redlands CA, USA). The base map of the global administrative areas was downloaded from the Natural Earth (https://www.naturalearthdata.com/).

\section{Results}

\section{Annual trends of malaria cases in Harari Region}

Within the period of $2013-2019$, a total of $44,882(46.9 \%)$ malaria cases were detected from 95,629 malaria-suspected outpatient diagnosed in the Harari Region with annual mean malaria cases of about 6,412 . Of these, 41,046 were microscopically and RDT confirmed malaria cases (annual mean $=5,864$ ) while 3,836 were reported as clinical cases in the Region. Despite malaria cases were reported in all years, there was a fluctuating trend of malaria within the study period. In 2016, the highest number of malariasuspected patients $(n=21,671)$ were examined and of them $11,605(53.6 \%)$ were confirmed malaria cases whereas the least number of cases (1,767 [12.5\%]), were reported in 2019 (Table 1). 
Table 1

Malaria suspected cases examined, parasitological confirmed and clinically identified malaria cases in Harari Region from 2013 to 2019

\begin{tabular}{|llllllll|}
\hline Year & $\begin{array}{l}\text { Malaria } \\
\text { suspected cases } \\
\text { examined }\end{array}$ & $\begin{array}{l}\text { Total malaria Cases } \\
\text { (Confirmed }+ \\
\text { Clinical) }\end{array}$ & $\%$ & $\begin{array}{l}\text { Confirmed } \\
\text { malaria } \\
\text { Cases }\end{array}$ & \% & $\begin{array}{l}\text { Clinical } \\
\text { malaria } \\
\text { Cases }\end{array}$ & $\%$ \\
\hline 2013 & 11502 & 10448 & 90.8 & 9069 & 86.8 & 1379 & 13.2 \\
\hline 2014 & 5330 & 4105 & 77.0 & 4074 & 99.2 & 31 & 0.8 \\
\hline 2015 & 9536 & 5981 & 62.7 & 5614 & 93.9 & 367 & 6.1 \\
\hline 2016 & 21671 & 11605 & 53.6 & 10680 & 92.0 & 925 & 8.0 \\
\hline 2017 & 18814 & 8712 & 46.3 & 7621 & 87.5 & 1091 & 12.5 \\
\hline 2018 & 14658 & 2264 & 15.5 & 2221 & 98.1 & 43 & 1.9 \\
\hline 2019 & 14118 & 1767 & 12.5 & 1767 & 100 & 0 & 0.0 \\
\hline Grand & 95,629 & 44,882 & 46.9 & 41,046 & 91.4 & 3,836 & 8.6 \\
\hline Total & & & & & & & \\
\hline
\end{tabular}

In general, there was a decreasing trend in a number of confirmed malaria cases from 2013 to 2014 . However, malaria cases showed an increment from 2014 to 2016 which was followed by a decreasing trend in a number of malaria cases from 2017 to 2019 (Fig. 2).

With regard to Plasmodium species, throughout the reviewed period the numbers of $P$. falciparum cases were dominant over $P$. vivax with the exception of the year 2014. Out of the total confirmed malaria cases, $P$. falciparum accounted for 28,393 (69.2\%) whereas $P$. vivax 12,576 (30.6\%) cases. Mixed $P$. falciparum/P. vivax infections were insignificant in the Region which accounted for only $0.2 \%$ of total confirmed malaria cases $(n=77)$ (Table 2$)$. 
Table 2

Seven-year malaria parasite species composition in Harari region from 2013 to 2019

\begin{tabular}{|lllll|}
\hline Year & $\begin{array}{l}\text { P. falciparum } \\
\mathbf{n}(\%)\end{array}$ & $\begin{array}{l}\text { P. vivax } \\
\mathbf{n}(\%)\end{array}$ & $\begin{array}{l}\text { Mixed } \\
\mathbf{n}(\%)\end{array}$ & Total confirmed cases (\%) \\
\hline 2013 & $5256(58)$ & $3780(41.7)$ & $33(0.3)$ & $9069(22.1)$ \\
\hline 2014 & $1961(48.1)$ & $2113(51.9)$ & $0(0.0)$ & $4074(9.9)$ \\
\hline 2015 & $3896(69.4)$ & $1704(30.3)$ & $14(0.3)$ & $5614(13.7)$ \\
\hline 2016 & $8352(78.2)$ & $2302(21.6)$ & $26(0.2)$ & $10680(26.0)$ \\
\hline 2017 & $5825(76.4)$ & $1792(23.5)$ & $4(0.1)$ & $7621(18.6)$ \\
\hline 2018 & $1518(68.3)$ & $703(31.7)$ & $0(0.0)$ & $2221(5.4)$ \\
\hline 2019 & $1585(89.7)$ & $182(10.3)$ & $0(0.0)$ & $1767(4.3)$ \\
\hline Grand Total & $28,393(69.2)$ & $12,576(30.6)$ & $77(0.2)$ & $41,046(100)$ \\
\hline
\end{tabular}

The seven-year plasmodium species trend in Harari region indicates $P$. vivax malaria showed the same trend, a drop in number of cases throughout all years. On the other hand, $P$. falciparum cases consistently increased from 2014 to 2016 and downward trend afterward (Fig. 3).

\section{Malaria Cases Variation In Between Districts Of Harari Region}

Regardless of the apparent variation of malaria trends in the study area, malaria cases occurred in almost every district of the Region. Based on year by year reported malaria cases, the highest cases were reported from the Jenella district followed by the Erer and Amir Nur districts in decreasing order. These districts experienced $79.4 \%(35,646 / 44,882)$ of the total malaria cases in the Region. Conversely, the least malaria cases were tested and detected in Abadir, Shenkor and Aboker districts (Table 3). 
Table 3

Malaria cases distribution within districts of Harari Region from 2013 to 2019

\begin{tabular}{|c|c|c|c|c|c|c|c|c|}
\hline \multirow[t]{2}{*}{ Districts } & \multicolumn{7}{|c|}{ Total Malaria Cases } & \multirow[t]{2}{*}{ Total Cases n (\%) } \\
\hline & 2013 & 2014 & 2015 & 2016 & 2017 & 2018 & 2019 & \\
\hline Abadir & 39 & 0 & 6 & 8 & 0 & 0 & 0 & $53(0.1)$ \\
\hline Aboker & 32 & 191 & 36 & 86 & 80 & 19 & 16 & $460(1.0)$ \\
\hline Amir Nur & 333 & 234 & 1552 & 3352 & 1790 & 220 & 164 & $7,645(17.0)$ \\
\hline Dire eyara & 108 & 147 & 281 & 227 & 159 & 57 & 32 & $1,011(2.3)$ \\
\hline Erer & 3831 & 1172 & 1161 & 1303 & 1565 & 314 & 127 & $9,473(21.1)$ \\
\hline Hakim & 584 & 360 & 590 & 1238 & 1039 & 395 & 658 & $4,864(10.8)$ \\
\hline Jenella & 5179 & 1910 & 2164 & 4751 & 3084 & 1049 & 391 & $18,528(41.3)$ \\
\hline Shenkor & 0 & 4 & 0 & 3 & 0 & 54 & 16 & $77(0.2)$ \\
\hline Sofi & 342 & 87 & 191 & 637 & 995 & 156 & 363 & $2,771(6.2)$ \\
\hline Total & 10,448 & 4,105 & 5,981 & 11,605 & 8,712 & 2,264 & 1,767 & $44,882(100)$ \\
\hline \multicolumn{9}{|c|}{ Sub-district (Kebele) level stratification of malaria incidence } \\
\hline \multicolumn{9}{|c|}{$\begin{array}{l}\text { Based on the API per } 1000 \text { population, one of the major determinates of malaria transmission which } \\
\text { used for stratification; the sub-districts of Harari Region stratified into three broad strata of malaria. } \\
\text { They are malaria-free }(\mathrm{API}=0) \text {, low- }(0<\mathrm{API}>5) \text { and moderate-transmission strata ( } 5 \leq \mathrm{API}>100) \\
\text { (Fig. 4). }\end{array}$} \\
\hline
\end{tabular}

In Harari Region according to the latest (2019) epidemiological data of the region's malaria stratification, $8.3 \%(3 / 36)$ of sub-districts reported no malaria and a majority (22 [61.1\%]) of sub-districts reported fewer than five cases per thousand population. These low malaria strata sub-districts accounted for $28.4 \%$ (257/906) of malaria cases. The moderate strata were found at 11 (30.6\%) sub-districts and accounted for $71.6 \%(649 / 906)$ of malaria cases. Furthermore, there were no high malaria strata in the Harari Region. Based on the updated map of the Harari Region the numbers of sub-districts and population size for defined categories of malaria strata have been presented as follows (Table 4). 
Table 4

Numbers of sub-districts and population size for defined categories of malaria strata (2019) in Harari Region

\begin{tabular}{|c|c|c|c|c|c|c|}
\hline $\begin{array}{l}\text { Malaria Strata } \\
\text { (Elimination Phase) }\end{array}$ & API & $\begin{array}{l}\text { Population } \\
\text { (2019) }\end{array}$ & $\begin{array}{l}\% \\
\text { Population }\end{array}$ & $\begin{array}{l}\text { Total } \\
\text { malaria } \\
\text { cases } \\
(2019)\end{array}$ & $\begin{array}{l}\text { Number of } \\
\text { Sub- } \\
\text { districts }\end{array}$ & $\begin{array}{l}\% \\
\text { Sub- } \\
\text { districts }\end{array}$ \\
\hline $\begin{array}{l}\text { Free } \\
\text { (Prevention of } \\
\text { reintroduction) }\end{array}$ & 0 & 21,230 & $8.1 \%$ & 0 & 3 & $8.3 \%$ \\
\hline $\begin{array}{l}\text { Low } \\
\text { (Elimination) }\end{array}$ & $\begin{array}{l}0< \\
\mathrm{API}>5\end{array}$ & 171,745 & $65.1 \%$ & 257 & 22 & $61.1 \%$ \\
\hline $\begin{array}{l}\text { Moderate } \\
\text { (Pre-Elimination) }\end{array}$ & $\begin{array}{l}5 \leq \\
\text { API> } \\
100\end{array}$ & 70,681 & $26.8 \%$ & 649 & 11 & $30.6 \%$ \\
\hline High (Optimization) & $\geq 100$ & 0 & $0.0 \%$ & 0 & 0 & $0.0 \%$ \\
\hline Total & & 263,656 & $100 \%$ & 906 & 36 & $100 \%$ \\
\hline
\end{tabular}

Based on the malaria positivity rate, one of the major indicators of malaria case burden, in Harari Region positivity rate has been reduced from $21.9 \%$ in 2017 to $10.6 \%$ in 2019. Although some of sub-districts were below $5 \%$, malaria test positivity rate is still greater than $10 \%$ in some of elimination sub-districts in the Region (Fig. 5).

\section{Seasonal Variation Of Malaria Cases In The Harari Region}

In the present study, despite the apparent fluctuation of malaria trends, cases occurred in almost every month of the year in the region. The highest peak of malaria cases in almost all years was observed from September to November. Correspondingly, there was a smaller peak of malaria cases from April to May. The minimum number of malaria cases were observed from December to February. At species level, the higher number of $P$. falciparum and $P$. vivax cases were diagnosed and identified from September to November whereas the lower number of the malaria parasites were observed from December to February (Fig. 6).

\section{Discussion}

The present study demonstrated the seven years annual trends, distribution and seasonal variation of malaria cases and Plasmodium species in Harari Region. In addition, it also shows malaria incidence stratification at sub-district level and variation of cases between districts. The study confirmed that malaria risk appears to be heterogeneous and varies greatly between districts. Based on the seven years retrospective data, malaria cases were higher in Jenella, Erer and Amir Nur districts and about $80 \%$ of the 
cases were from these districts. Furthermore, API-based stratification indicates that $70 \%$ of sub-districts reported fewer than five cases per thousand population. The findings from this study may support the designing of the large-scale malaria interventions and resource mobilization for malaria elimination in the Region.

The number of malaria cases in the study area showed year-to-year heterogeneous trend over the period of 2013 to 2019. A year by year report indicates the higher malaria-suspected patients and confirmed malaria cases in the Region were observed in 2016. This could be due to a number of factors such as, climatic changes and attendant irregularities in rainfall and temperature, and increased agricultural activities such as irrigation in the study area [14]. In contrary, there was a decrease in malaria cases from the year 2017 to 2019. This reduction in malaria cases in the Region might be attributed to the increased awareness of the community towards the application of different malaria control measures such as insecticides and repellents, frequent use of insecticide-treated nets and increased coverage of IRS. Moreover, since the launching of malaria elimination program in the Harari Region there is increased attention to scaling up malaria control and preventive activities by all stakeholders [8].

In the study area, $P$. falciparum was the dominant species followed by $P$. vivax. With the exception of the year $2014 P$. falciparum was the leading malaria parasite species in the study area. This is in agreement with other recent study reports from different parts of Ethiopia $[15,16,17]$. The finding was also in line with the national profile of malaria parasite distribution in Ethiopia which indicates that $P$. falciparum and $P$. vivax are the two dominant malaria parasites, distributed all over the country $[5,18]$. In contrast, a retrospective data from different parts of Ethiopia indicated that almost equal distribution of $P$. falciparum and $P$. vivax [19].

In Harari Region, based on the reported malaria cases, the highest number of cases were reported from the Jenella followed by Erer and Amir Nur districts. These districts bore $80 \%$ of the total malaria cases in the Region. These differences in malaria cases might be due to differences in climate, topography and malaria diagnostic techniques used. The Jenella district is one of the oldest known malaria diagnostic centre in the Region that most of the febrile cases from the Region comes to the Jenella Health centre to be tested for malaria cases which may increase the cases in the district. In Erer district there is seasonal Erer River which originate in the highlands and crosses the district. The River has high potential of irrigation and the farmers in the district use the water for irrigation along the cost. This seasonal river may create some puddles around the shore and irrigated villages could be the potential breeding sites to Anopheles mosquitoes. High larvae and adult vector and a higher prevalence of malaria were registered in the irrigated village, this could contribute for malaria transmission in the area [20,21]. Thus, clarifying the heterogeneous nature of malaria transmission at the district level in the Region is important in developing dynamic and area-specific risk maps to identify locations and populations at highest risk for appropriate planning, implementation of targeted and epidemiologically sound preventive and control measures against the disease [21,22]. Furthermore, there is also frequent human movement from Region to Region particularly from neighbouring malaria endemic areas of the Oromia and Somali Regions 
which may contribute for the increment of imported malaria cases in these districts that may challenge the malaria elimination from the Region.

The sub-districts level stratification (malaria-free, low, and moderate and high transmission) of malaria risk based on the three-year annual parasite incidence (API) per 1000 population showed that through the period of 2017-2019 there was expansion of malaria-free and the low transmission stratum. The number of sub-districts reclassified from moderate stratum to low were keep increasing from 2017 to 2019. This may be attributed to the impact of the ongoing malaria control interventions coverage, and increased community awareness and participation which updated the Region malaria map. This is in line with the current national malaria picture that demonstrate further shrinking of malaria in the Region [5].

In the Harari Region, a seven-year data revealed that the high malaria cases almost in all year was recorded from September to November, followed by April to May. These two seasons are the major and minor malaria transmission periods in Ethiopia, respectively. The major malaria transmission period follows the heavy rains from June to August which create a suitable environment for the breading of Anopheles mosquitoes. The minor transmission season occurs between April and May following the small shower of rains from February to March. These peak malaria seasons in study area are similar with other studies from different parts of the country $[15,16,17]$. This result may suggest that malaria control interventions such as IRS in the study area should be designed based on the malaria transmission patterns.

This analysis used the malaria data of over seven years period at Regional level and three years data at sub-district level. Nonetheless there are some limitations to the analysis, for API-based stratification of the malaria cases this paper cannot find the sub-district level shape file of Harar Town. However, the API of these sub-districts were $<1$, therefore the current analysis aggregated all cases reported from these sub-districts as malaria cases of Harar Town and presented in a table format for further clarification. The Region has relatively high health services coverage and patients from neighbouring Regions (Somali and Oromia) utilize health services in the Region. Therefore, the number of malaria cases reported here might not necessarily reflect the number of cases in the Region. Nonetheless, the analysis assumes that only people with severe complications of malaria might seek health services particular in the hospitals in Harari Region and those are often small in number.

\section{Conclusions}

In conclusion, after the introduction of current malaria control and elimination strategies malaria cases showed a significant decline in the Harari Region. Malaria transmission is ubiquitous in the Region, heterogeneous in the distribution and seasonal in pattern of number of cases. Over the period of seven years the burden of malaria significantly decreased. If this achievement is sustained, elimination of the disease from the study area might be feasible. However, there is apparent variation of malaria trends between districts and sub-districts. Therefore, there is a need for intensifying the existing malaria 
prevention and control strategies in the Region by focusing on those populations living in the higher malaria transmission districts and sub-districts.

\section{Abbreviations}

API: Annual parasite incidence; HMIS: Health management information system; IRS: Indoor residual spraying; SOPs: Standard operating procedures; WHO: World health organization.

\section{Declarations}

\section{Acknowledgements}

The authors are grateful to the Abadir, Aboker, Amir Nur, Dire Teyara, Jenella, Sofi, Erer, Hakim and Shenkor District Health offices and Harari Regional Health Bureau for their cooperation during data collection. In addition, many thanks are owed to the Harari Regional Health Bureau malaria control and elimination team for their cooperation by providing necessary facilities during data collection and for their technical support.

\section{Authors' contributions}

The study design was conceived by EE, FM, SY and KD. Planning and implementation of the study, data collection and entry were conducted by EE, AT, AA, II, DD and EAB. Data analysis was conducted by EE, AT and KD; drafted the manuscript: EE and KD; critically commented on the manuscript: EE, FM, SY and KD. All authors read and approved the final manuscript.

\section{Funding}

The authors did not receive funding for the current study. KD is supported by the Well come Trust [grant number 201900/Z/16/Z] as part of his International Intermediate Fellowship.

\section{Availability of data and materials}

Data supporting the conclusions and outcomes of this article are included within the article. The raw datasets presented and analyzed in this study are available upon request from the corresponding author.

\section{Ethics approval and consent to participate}

This study was conducted after discussing the aim and the information required for the study and official letter was obtained from Harari Regional Health Bureau. Written permission was also obtained from the head of each district Health office using official letters before data collection.

\section{Consent for publication}

Not applicable. 


\section{Competing interests}

The authors declare that they have no competing interests.

\section{Author details}

${ }^{1}$ Harari Regional Health Bureau, Malaria Control and Elimination Program, Harar, Ethiopia; ${ }^{2}$ Malaria and Neglected Tropical Diseases Research Directorate, Armauer Hansen Research Institute, Addis Ababa, Ethiopia; ${ }^{3}$ Arba Minch University, Department of Biology, Arba Minch, Ethiopia; ${ }^{4}$ Federal Ministry of Health, Addis Ababa, Ethiopia; ${ }^{5}$ Jigjiga University, Jigjiga, Ethiopia; ${ }^{6} \mathrm{Global}$ Health and Infection Department, Brighton and Sussex Medical School, Brighton, BN 19PX, UK; ${ }^{7}$ School of Public Health, College of Health Sciences, Addis Ababa University, Addis Ababa, Ethiopia

\section{References}

1. Murray CJL, Ortblad KF, Guinovart C, Lim SS, Wolock TM, Roberts DA, et al. Global, regional, and national incidence and mortality for HIV, tuberculosis, and malaria during 1990-2013: a systematic analysis for the Global Burden of Disease Study 2013. Lancet. 2014;384:1005-70.

2. World Health Organization. World Malaria Report. Geneva: World Health Organization; 2019.

3. Aregawi M, Lynch M, Bekele W, Kebede H, Jima D, Tafese $H$, et al. Time series analysis of trends in malaria cases and deaths at hospitals and the effect of antimalarial interventions, 2001-2011, Ethiopia. PLOS ONE. 2014;9:e106359.

4. Federal Ministry of Health. National Malaria Strategic Plan 2014-2020. Addis Ababa: Federal Ministry of Health; 2014.

5. Federal Ministry of Health. National Malaria Strategic Plan 2017-2020. Addis Ababa: Federal Ministry of Health; 2017.

6. Tesfaye S, Belyhun Y, Teklu T, Mengesha T, Petros B. Malaria prevalence pattern observed in the highland fringe of Butajira, Southern Ethiopia: A longitudinal study from parasitological and entomological survey. Malaria Journal. 2011;10:153.

7. Daygena TY, Massebo F, Lindtjørn B. Variation in species composition and infection rates of Anopheles mosquitoes at different altitudinal transects, and the risk of malaria in the highland of Dirashe Woreda, south Ethiopia. Parasites Vectors. 2017;10:343.

8. Federal Ministry of Health. National malaria elimination road map. Addis Ababa: Federal Ministry of Health; 2017.

9. Abeku TA, Helinski MEH, Kirby MJ, Kefyalew T, Awano T, Batisso E, et al. Monitoring changes in malaria epidemiology and effectiveness of interventions in Ethiopia and Uganda: beyond Garki project baseline survey. Malaria Journal. 2015;14:337.

10. Federal Ministry of Health. Malaria diagnosis and treatment guidelines for health workers in Ethiopia. Addis Ababa: Federal Ministry of Health; 2004. pp. 1-57. 
11. World Health Organization. Disease surveillance for malaria control: an operational manual. Geneva: World Health Organization; 2012.

12. Federal Ministry of Health. Health Sector Development Programme IV (2010/11-2014/15). Addis Ababa: Federal Ministry of Health; 2010.

13. World Health Organization. Guidelines for the treatment of malaria. Thrid edn. Geneva, Switzerland: World Health Organization; 2015.

14. Reiter P. Climate change and mosquito borne diseases. Environ Health Perspect. 2001;109(suppl 1):141-61.

15. Alemu A, Muluye D, Mihret M, Adugna M, Gebeyaw M. Ten year trend analysis of malaria prevalence in Kola Diba, North Gondar, Northwest Ethiopia. Parasites Vectors. 2012;5:173.

16. Gebretsadik D, Feleke DG, Fiseha M. Eight-year trend analysis of malaria prevalence in Kombolcha, South Wollo, north-central Ethiopia: a retrospective study. Parasites Vectors. 2018;11:55.

17. Feleke DG, Gebretsadik D, Gebreweld A. Analysis of the trend of malaria prevalence in Ataye, North Shoa, Ethiopia between 2013 and 2017. Malaria Journal. 2018;17:323.

18. Ethiopian Public Health Institute. Ethiopia national malaria indicator survey 2015. Addis Ababa: Ethiopian Public Health Institute; 2016.

19. Argaw M, Woldegiorgis A, Abate D, Abebe ME. Improved malaria case management in formal private sector through public private partnership in Ethiopia: retrospective descriptive study. Malaria Journal. 2016;15:352.

20. Kibret S, Alemu Y, Boelee E, Tekie H, Alemu D, Petros B. The impact of a small-scale irrigation scheme on malaria transmission in Ziway area, central Ethiopia. Tropical Medicine International Health. 2010;15(1):41-50.

21. Esayas E, Woyessa A, Massebo F. Malaria infection clustered into small residential areas in lowlands of southern Ethiopia. Parasite Epidemiology Control. 2020;10:e00149.

22. Loha E, Lunde T, Lindtjørn B. Effect of Bed nets and Indoor Residual Spraying on Spatio-Temporal Clustering of Malaria in a Village in South Ethiopia: A Longitudinal Study. PLOS ONE. 2012;7:e47354.

\section{Figures}




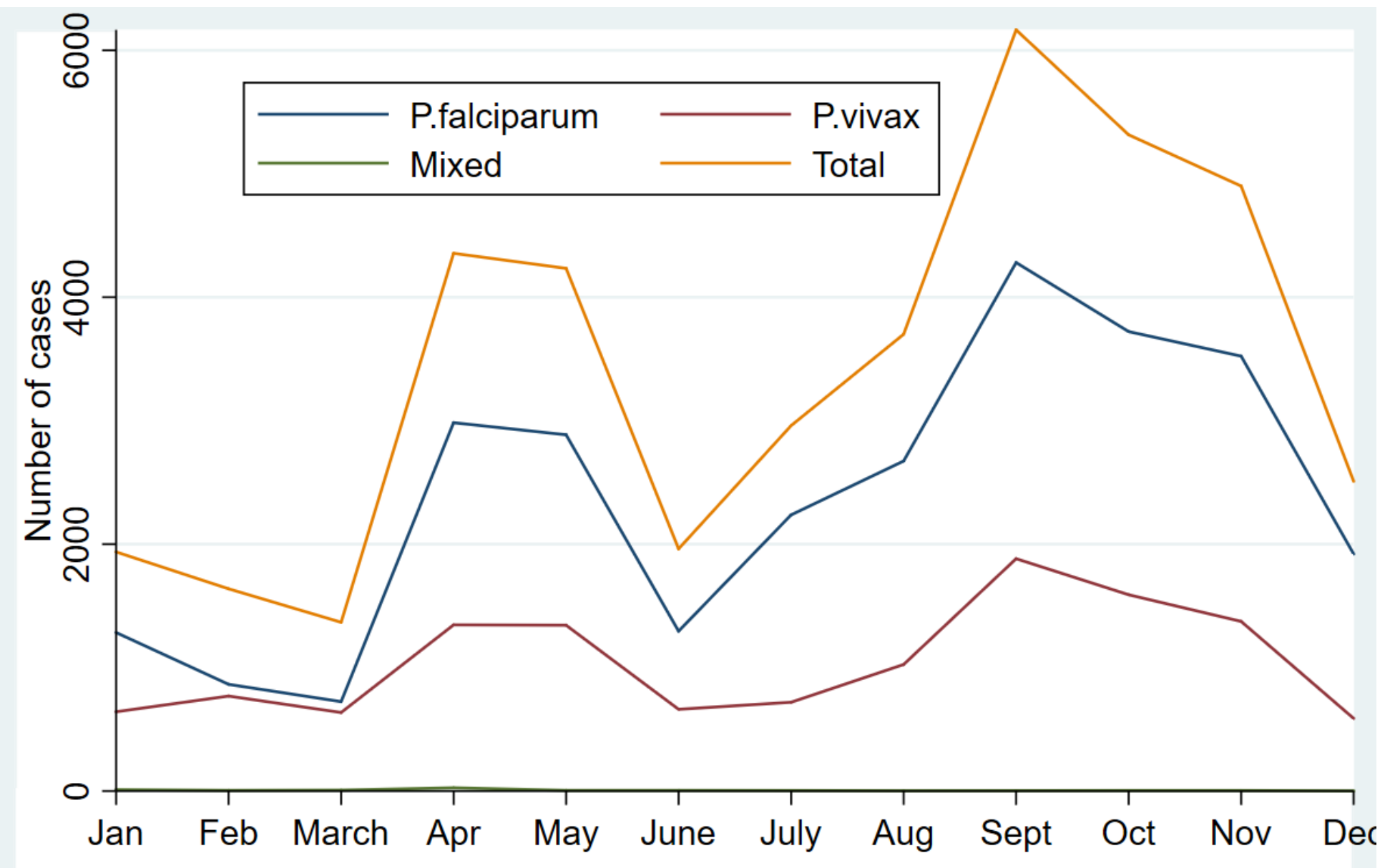

Figure 1 

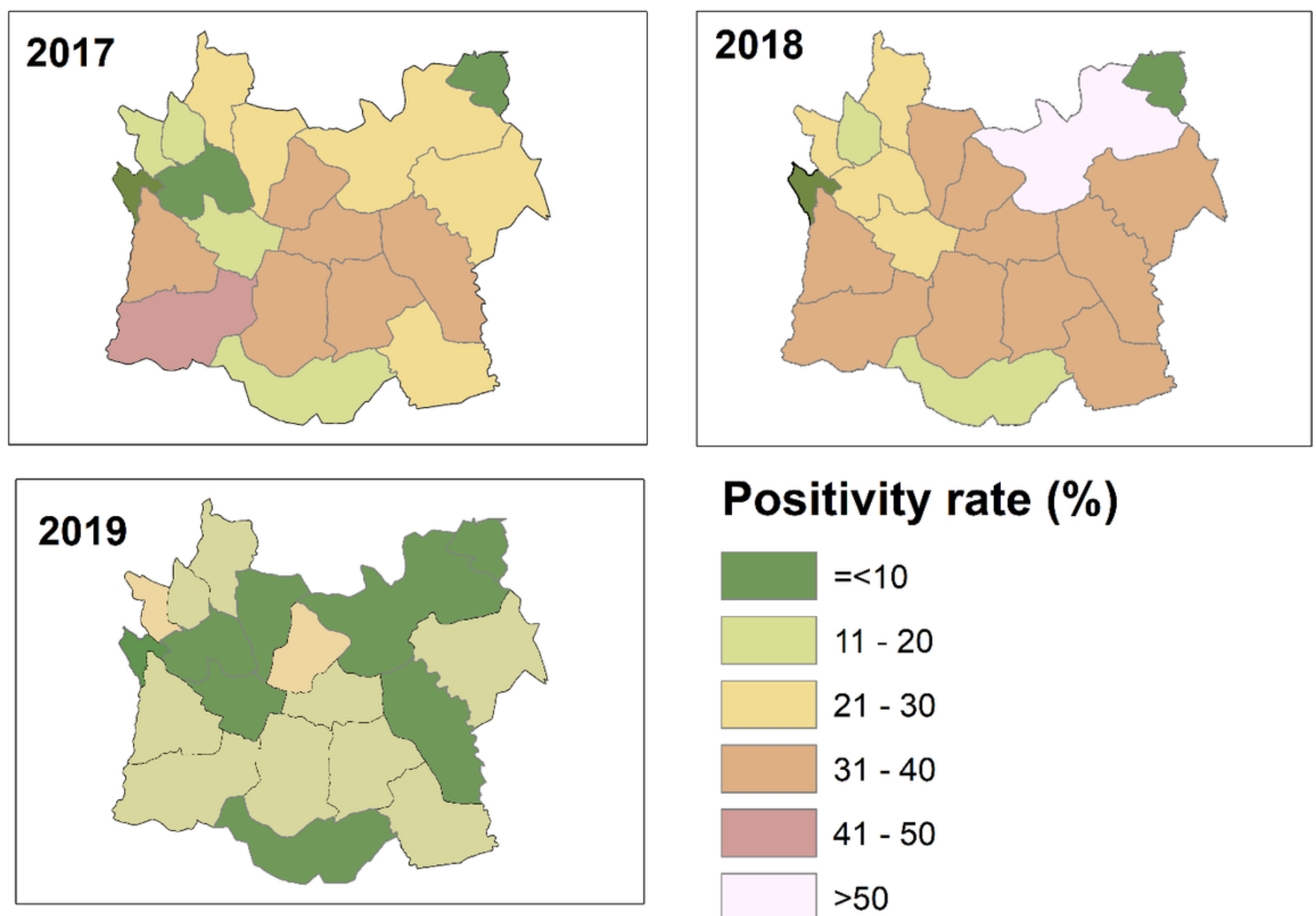

\section{Positivity rate (\%)}

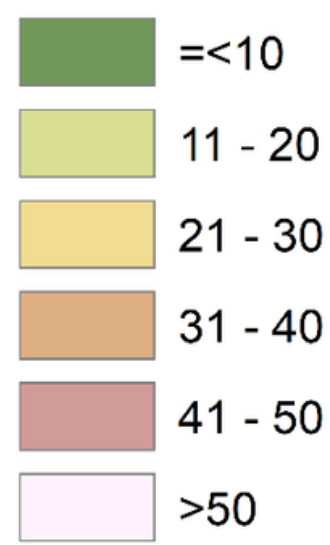

Figure 2 

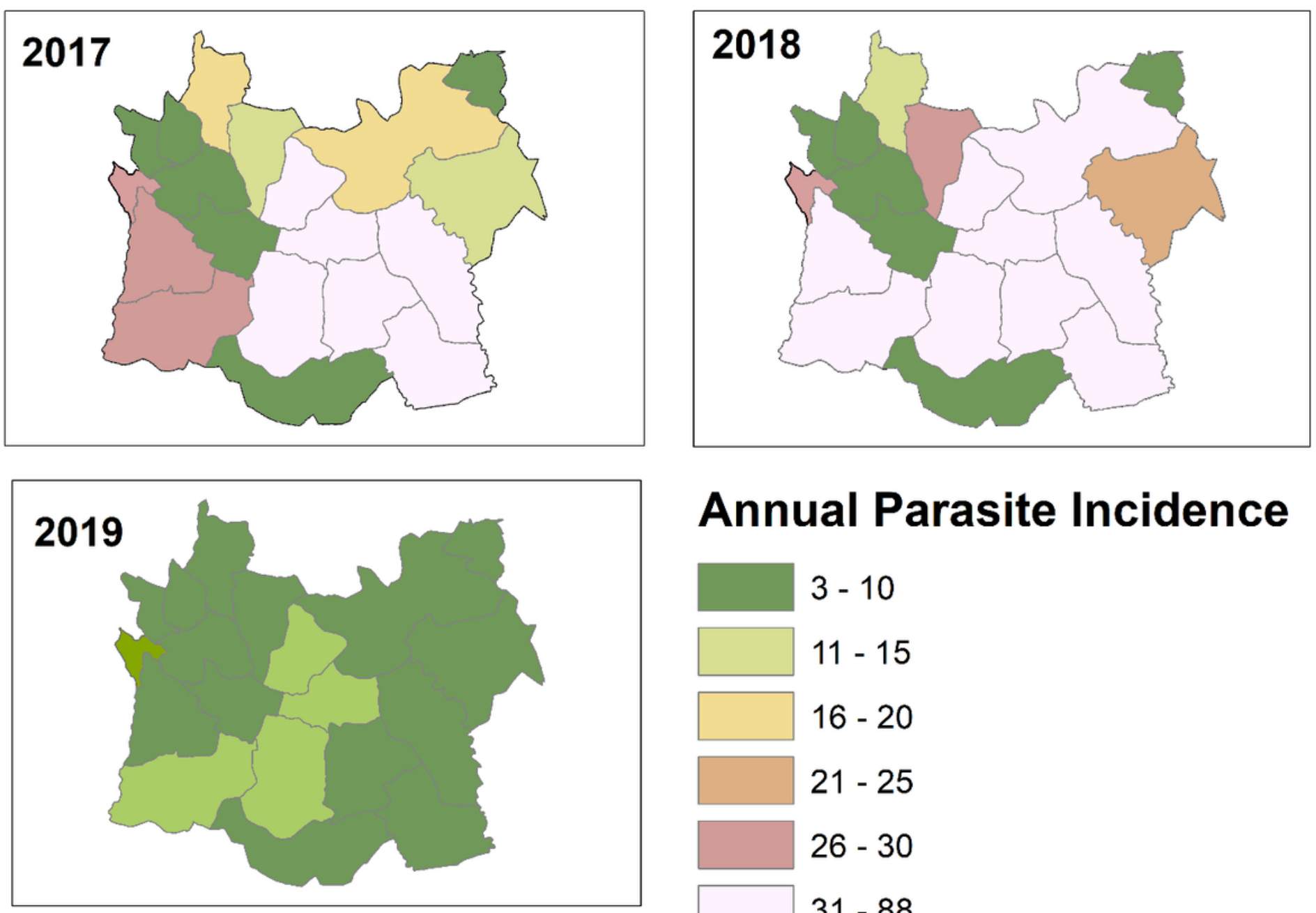

Annual Parasite Incidence

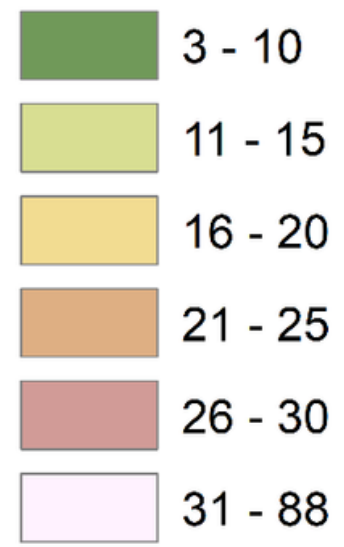

Figure 3 


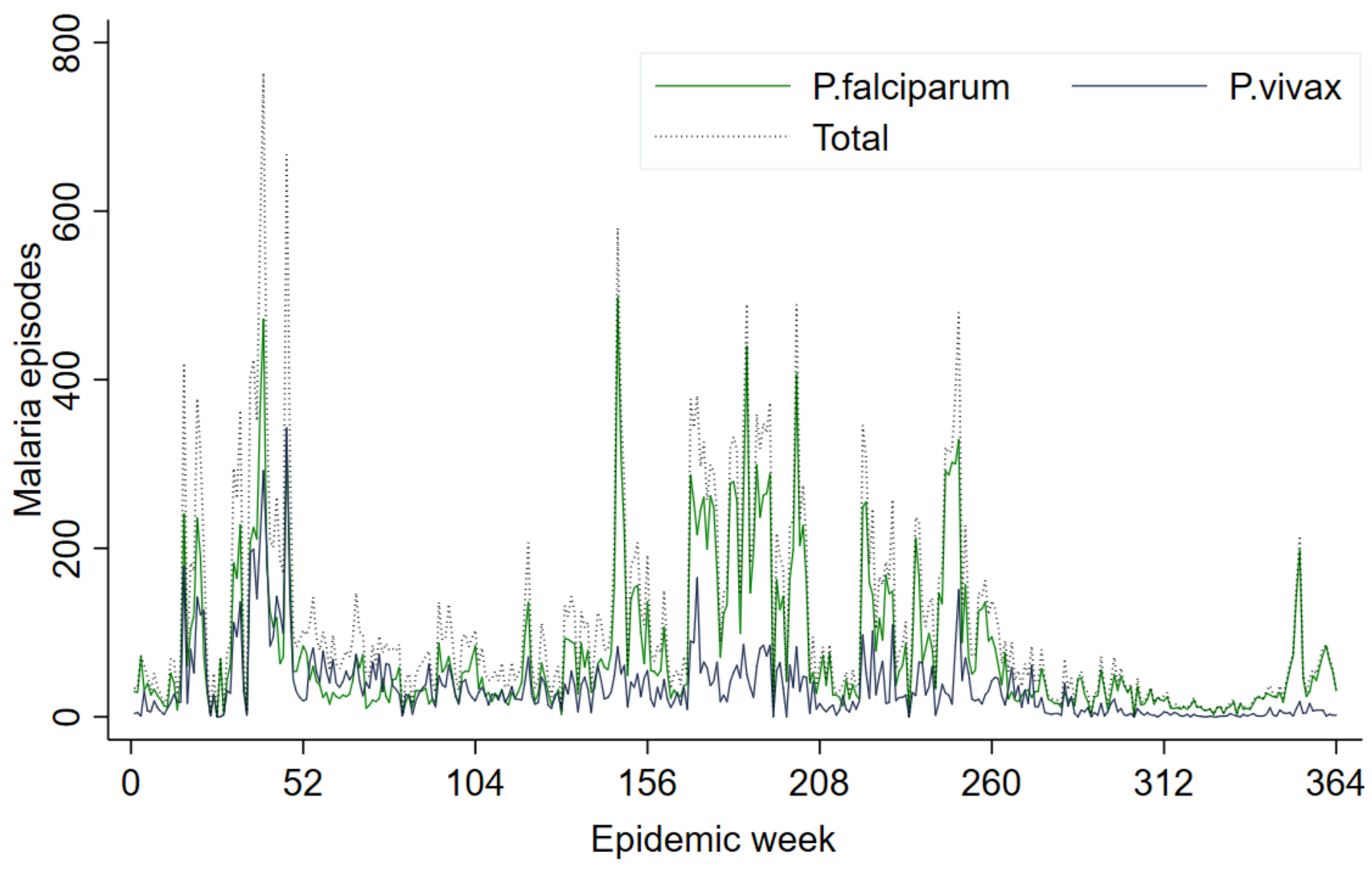

Figure 4 


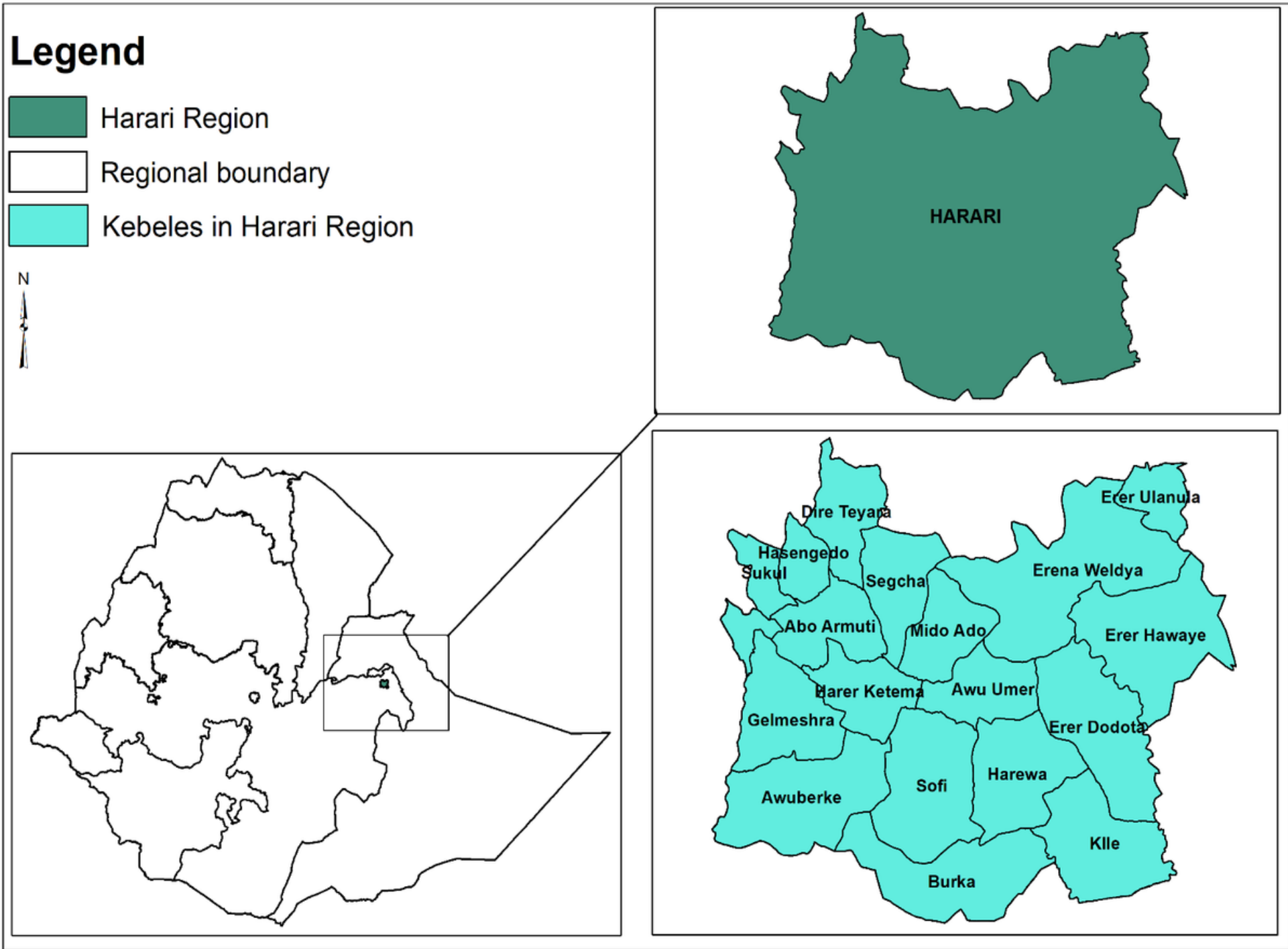

Figure 5

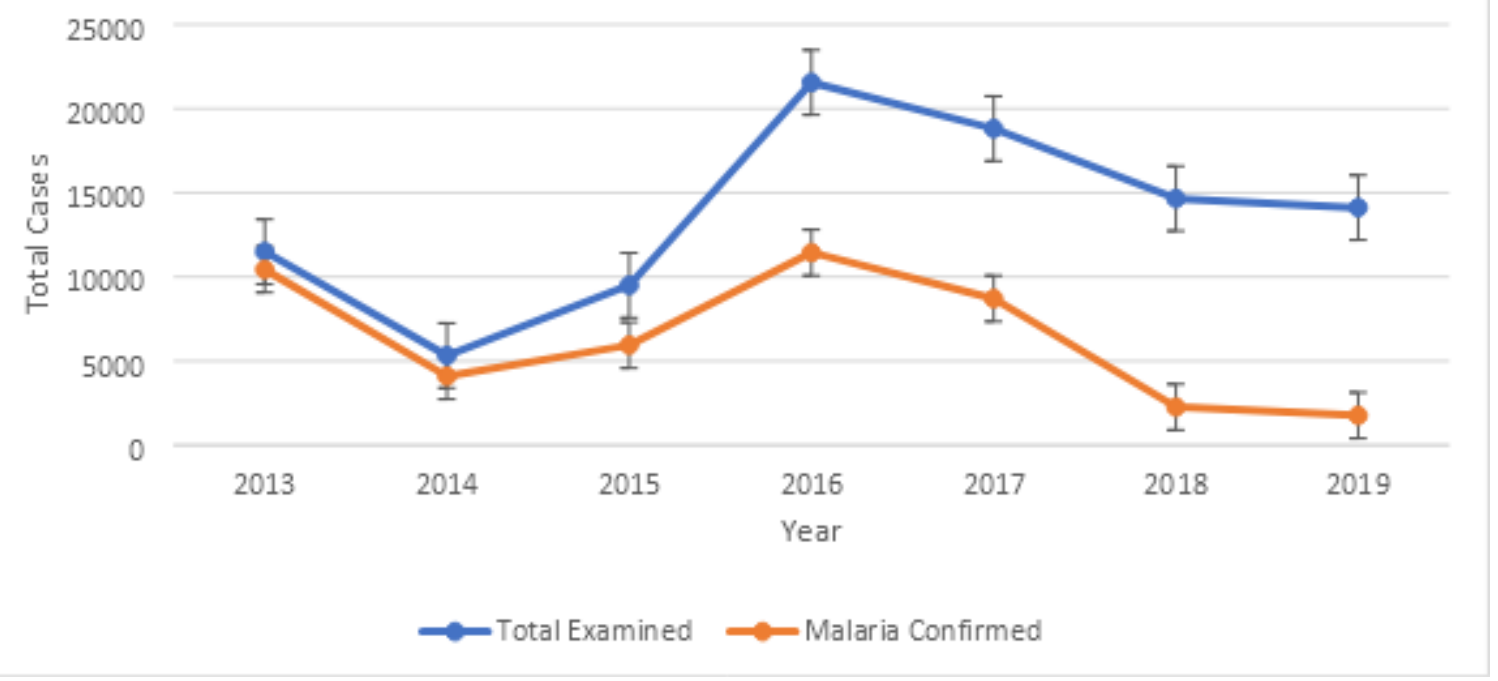

Figure 6 


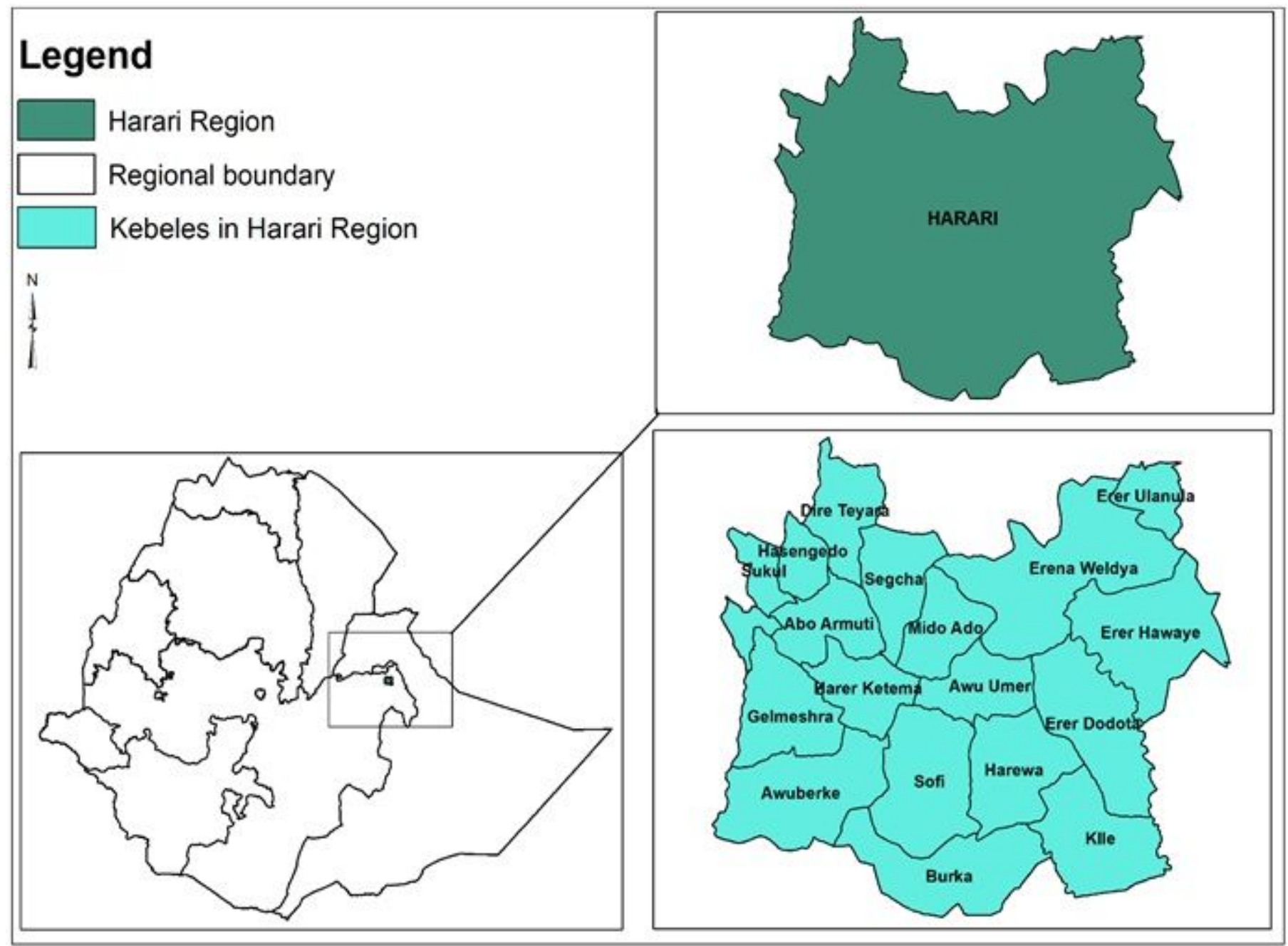

Figure 7

Location of Harari Region in map of Ethiopia and study sub-districts (kebeles).

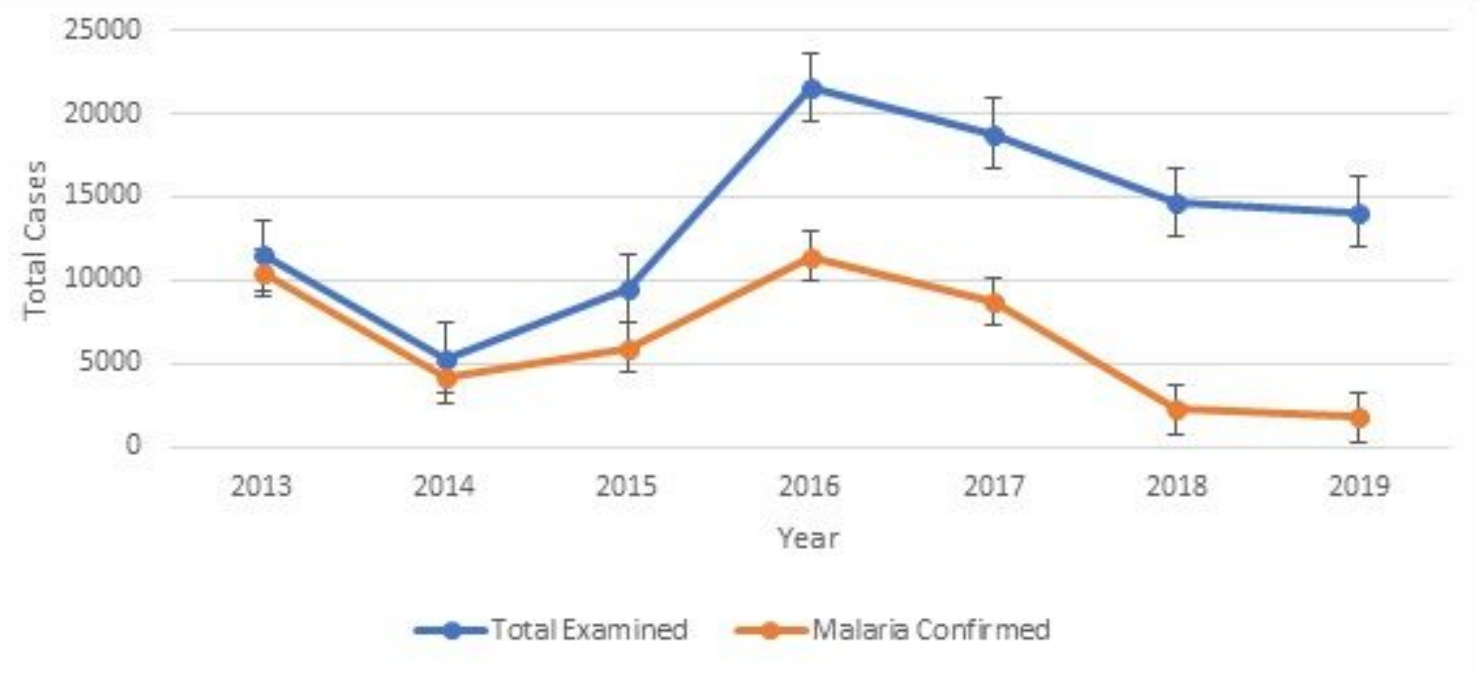

Figure 8

Annual trends of malaria cases in Harari region from 2013 to 2019 


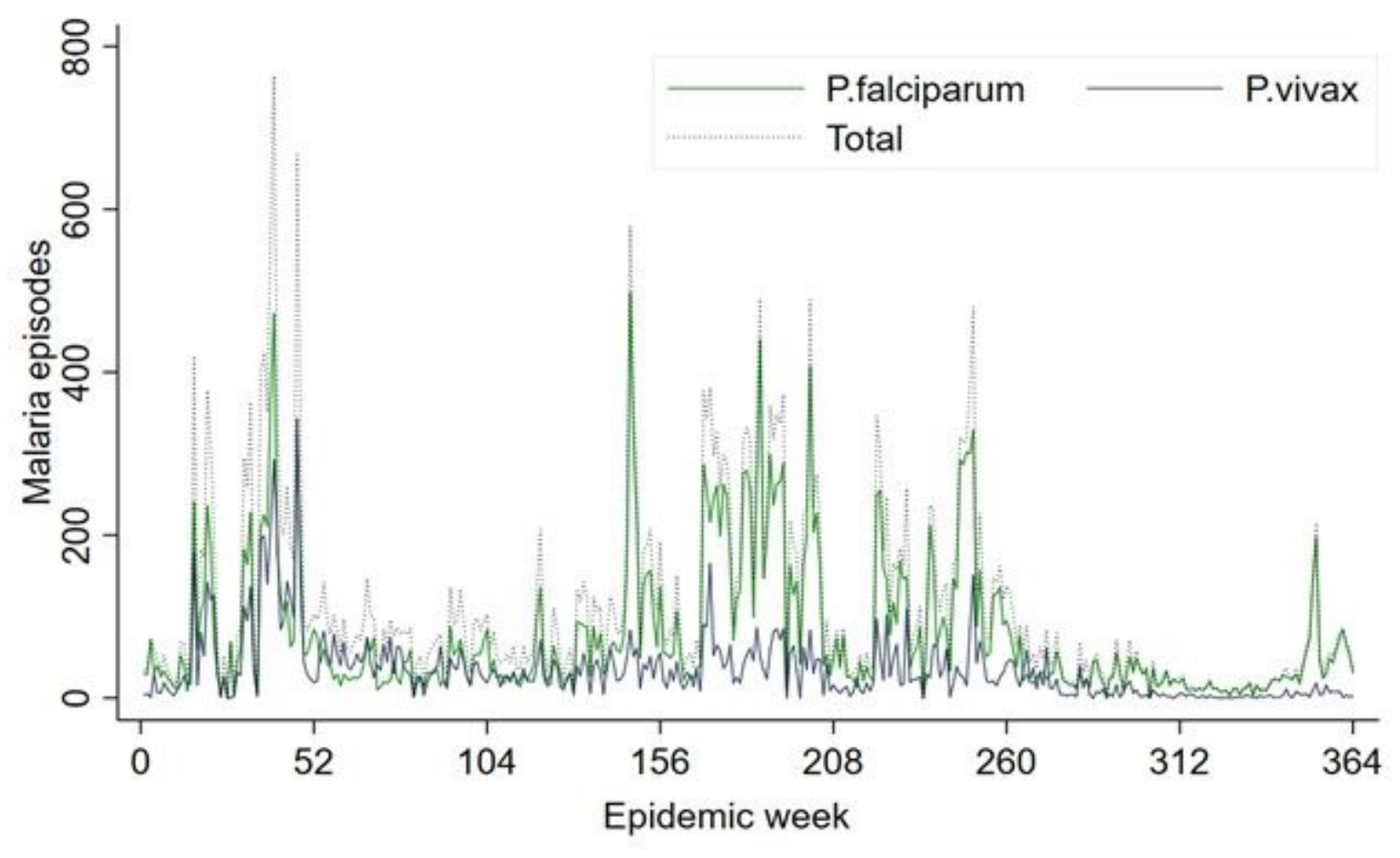

Figure 9

Weekly Plasmodium species trend in Harari region from 1st January 2013 to 31st December 2019
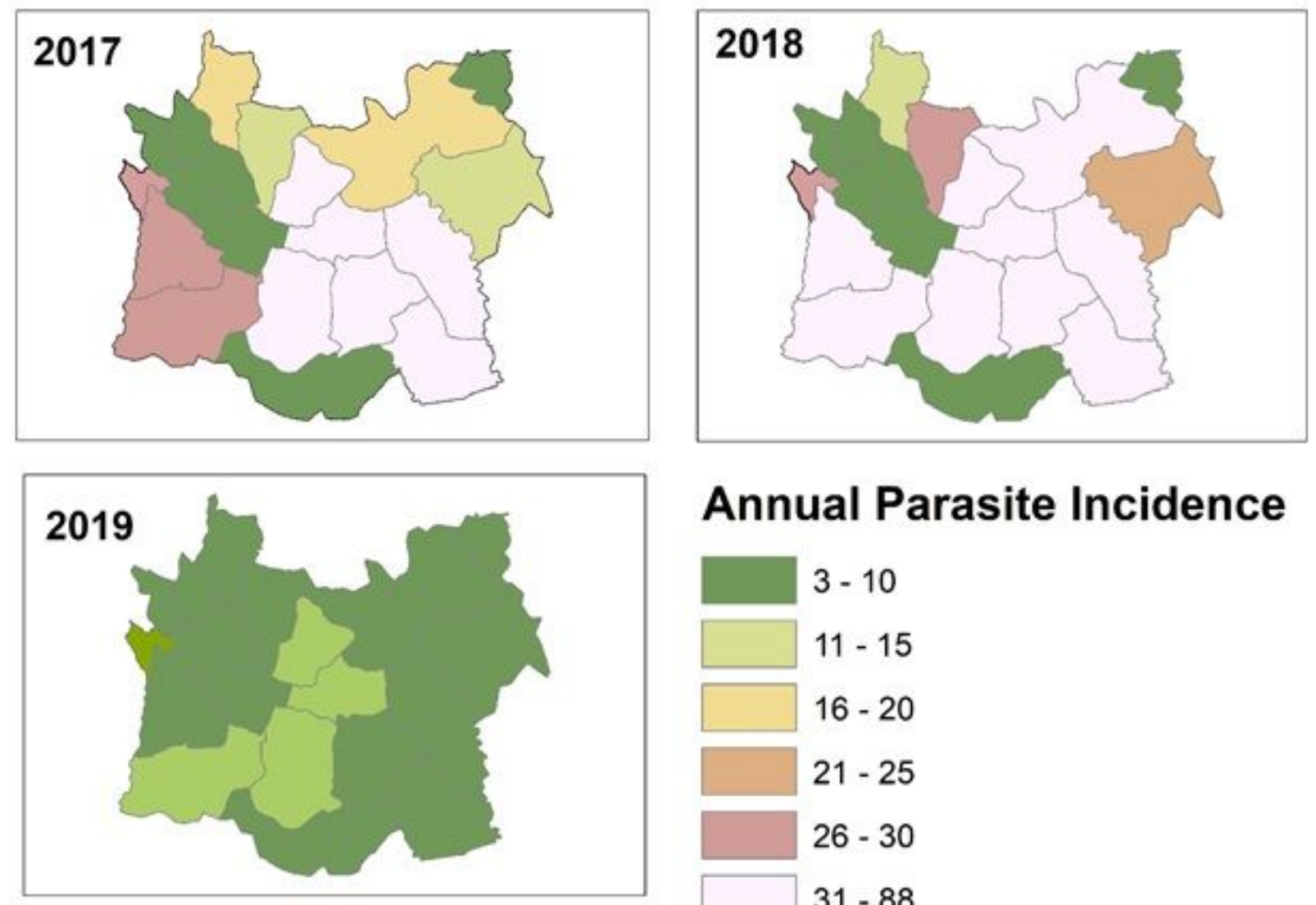

\section{Annual Parasite Incidence}

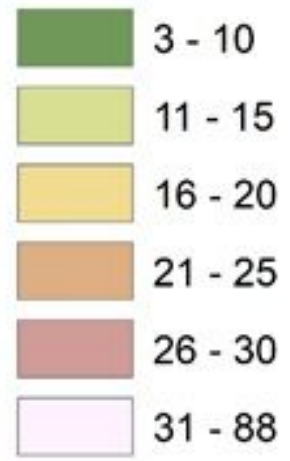

Figure 10 
Sub-districts level stratification of malaria incidence from 2017 to 2019, Harari region, Ethiopia
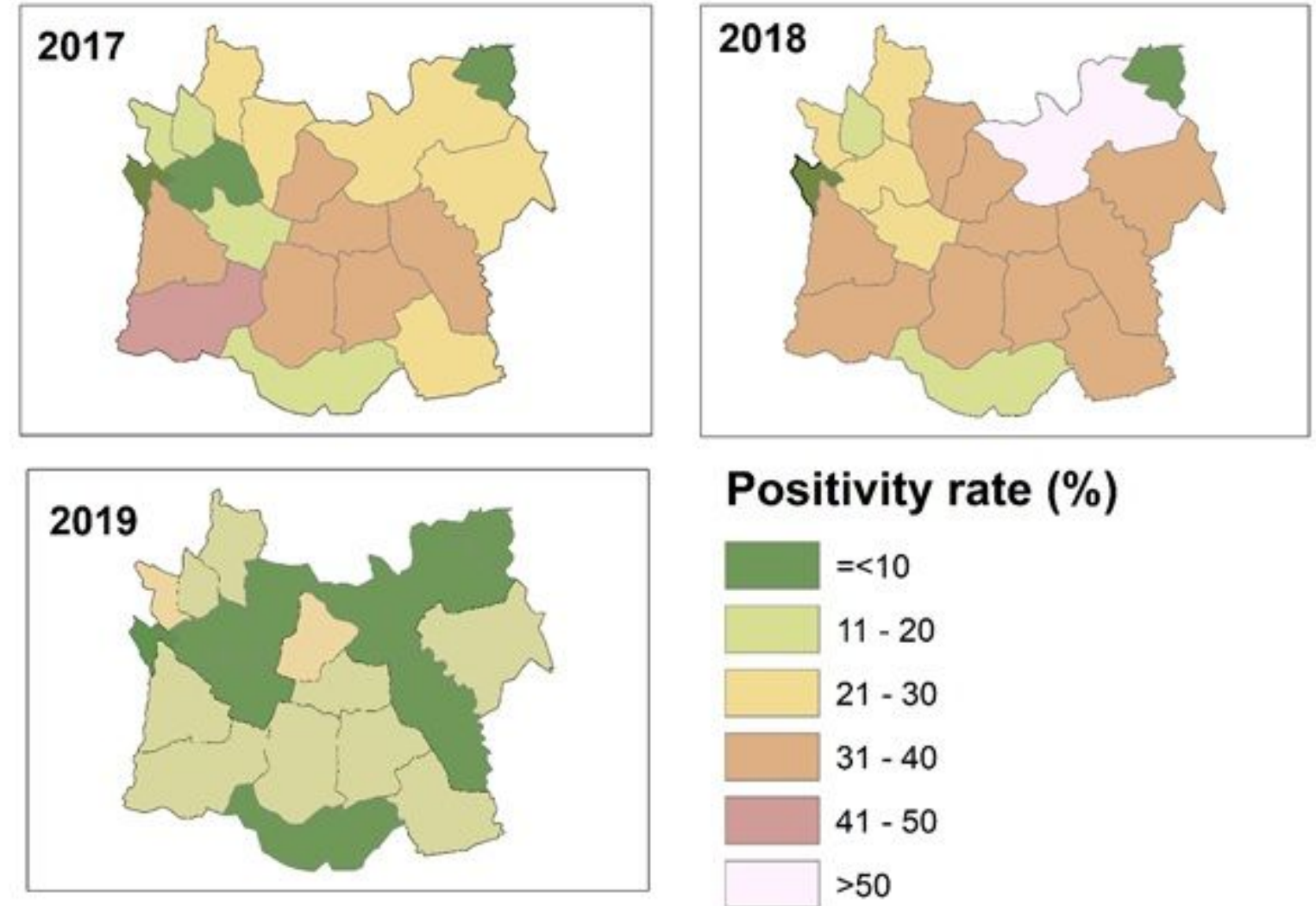

\section{Positivity rate (\%)}

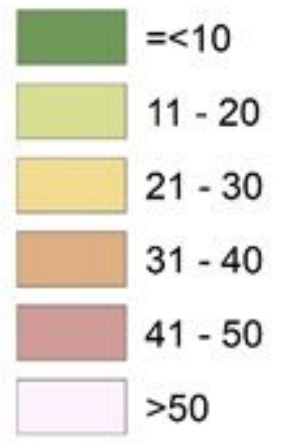

\section{Figure 11}

Sub-district level malaria positivity rate from 2017 to 2019, Harari Region, Ethiopia

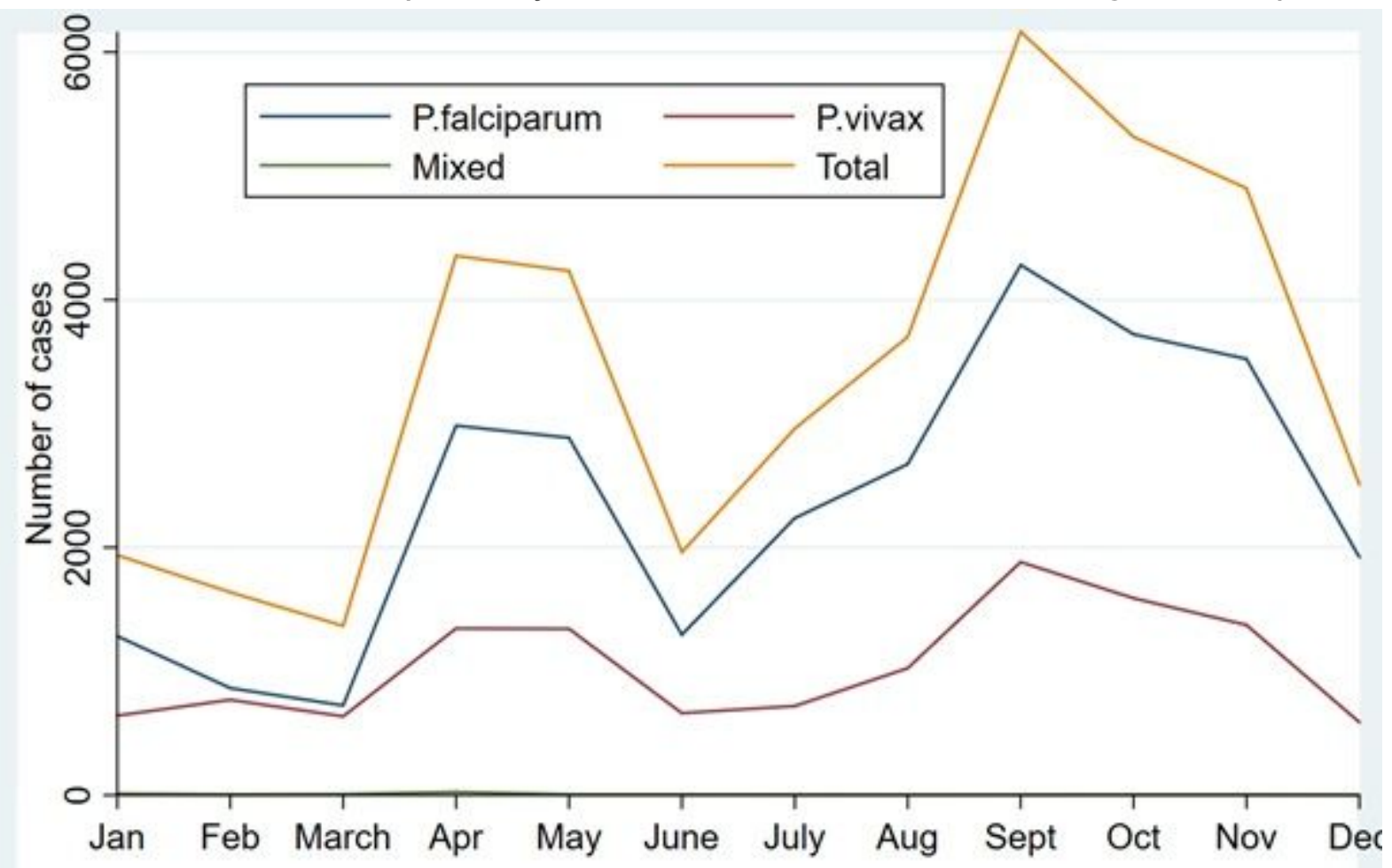

Figure 12 
Monthly distribution of Plasmodium species and total confirmed malaria cases in Harari Region, from January 2013 to December 2019

\section{Supplementary Files}

This is a list of supplementary files associated with this preprint. Click to download.

- supplement4.docx 